\title{
Dynamic Artificial Neural Network-based reliability considering operational context of assets.
}

\author{
J. Izquierdo ${ }^{\mathrm{a}, \mathrm{b}, * *}$, A. Crespo Márquez ${ }^{\mathrm{b}, *}$, J. Uribetxebarria ${ }^{\mathrm{a}}$ \\ ${ }^{a}$ IK4-Ikerlan Technology Research Centre, Operations and Maintenance Technologies Area, 20500 Gipuzkoa, Spain \\ ${ }^{b}$ Industrial Organization and Business Management I, School of Engineering, University of Seville, Camino de los \\ Descubrimientos $s / n, 41092$ Seville, Spain
}

\begin{abstract}
Assets reliability is a key issue to consider in the maintenance management policy and given its importance several estimation methods and models have been proposed within the reliability engineering discipline. However, these models involve certain assumptions which are the source of different uncertainties inherent to the estimations. An important source of uncertainty is the operational context in which the assets operate and how it affects the different failures. Therefore, this paper contributes to the reduction of the uncertainty coming from the operational context with the proposal of a novel method and its validation through a case study. The proposed model specifically addresses changes in the operational context by implementing dynamic capabilities in a new conception of the Proportional Hazards Model. It also allows to model interactions among working environment variables as well as hidden phenomena thanks to the integration within the model of artificial neural network methods.
\end{abstract}

Keywords: Dynamic Reliability, Proportional Hazards Model, Artificial Neural Networks, Operational Context, Maintenance Management, Epistemic Uncertainty

\section{Introduction}

With the emerging of engineered systems in the 19th century so did arise the need for a scientific discipline dealing with their reliability, this discipline began to be known as reliability engineering in the 1950s [1]. The assumptions under which traditional techniques of reliability were developed, simplify many of the real-life boundary conditions; the motivation lying behind these simplifications, was the pressure of upholding the rigid and fast technological advances in the industries which initially showed more interest in reliability engineering (maritime, military, aircraft and Oil \& Gas industries) [2].

As the independent scientific discipline that reliability engineering has become, it measures the reliability by quantitative metrics and controls throughout the product lifecycle [3]. According to Zio [1], the purpose of reliability engineering is to provide a collection of formal methods aiming at exploring the relation among system operation and failure, and it is in this discipline where this paper intends to contribute with the novel model later on proposed.

\subsection{Motivation and research}

Reliability is a key issue to address by comprehensively analyzing the asset performance under the effects of different uncertainties during its life cycle [4]. The uncertainties affecting the asset performance have generally been classified into two types (see [3-7]):

- Aleatory uncertainty. Considered to be the result of a fundamental randomness in the natural phenomena of the world, it results in uncertainty referring to the inherent physical behavior of the system.

- Epistemic uncertainty. Associated with the lack of knowledge, it refers to the uncertainty related to the completeness and accuracy in the understanding of the failure process.

\footnotetext{
*Principal corresponding author, Tel.: +34 954487215.

** Corresponding author, Tel.: +34 943712400.

Email addresses: jizquierdo@ikerlan.es (J. Izquierdo), adolfo@etsi.us.es (A. Crespo Márquez), juribetxebarria@ikerlan.es (J. Uribetxebarria)
} 
As stated before, traditional reliability models involve assumptions and simplifications[8]; these are the sources of significant uncertainties causing the inability of the reliability models to properly describe the true behavior of the system [5].

Some important assumptions that introduce epistemic uncertainty in the models are the independence among components, the renewal assumption o the constant operational condition and external factors [9]. Such premises and suppositions are the cause of epistemic uncertainty, reducible by integrating new information on the contrary of aleatory uncertainty, which comes from the stochastic character of the problem [10]. Therefore, if the models-based methods aim at accurately quantify the reliability, it is a requisite for them to integrate the effect of epistemic uncertainties [3]. It is within this motivation where the scope of the paper lies; more precisely, the authors have developed a novel model for reliability estimation that reduces epistemic uncertainty referring to operational conditions of the assets.

A more realistic estimation of reliability, through a model integrating operating environment information, will enable more effective and better-customized maintenance policies [11]. The benefits of such models gain special interest in the early stages of the asset life-cycle, where it is relevant to reflect the context into which they will perform [12]. This aspect is a key pillar to take into account when designing the maintenance strategy, and it has been addressed throughout the later on reviewed literature (see Subsection 1.2).

It is this main pillar which gives birth to the research process conducted in this paper and summarized in figure 1. The conducted research, aiming at integrating the operational context effect on reliability assessment, have followed two courses; in the last phase, they converge into a comprehensive model that gathers the benefits from the researches regarding both lines. These research lines face two problems derived from the main motivation. The research represented in the bottom line aims at modeling the effects of changing operational environment, it deals with the reality of the same asset performing in very different environments and stress levels during its life-cycle. However, the research course represented by the top line of the process deals with the problem of modeling unknown interactions among the different parameters of the operational environment of the asset. Finally, the convergence of both lines, hence the merge of two innovative models, leads to the proposal of a novel model which not only integrates the dynamism of operating environments but the possible unknown interactions among its variables as well.

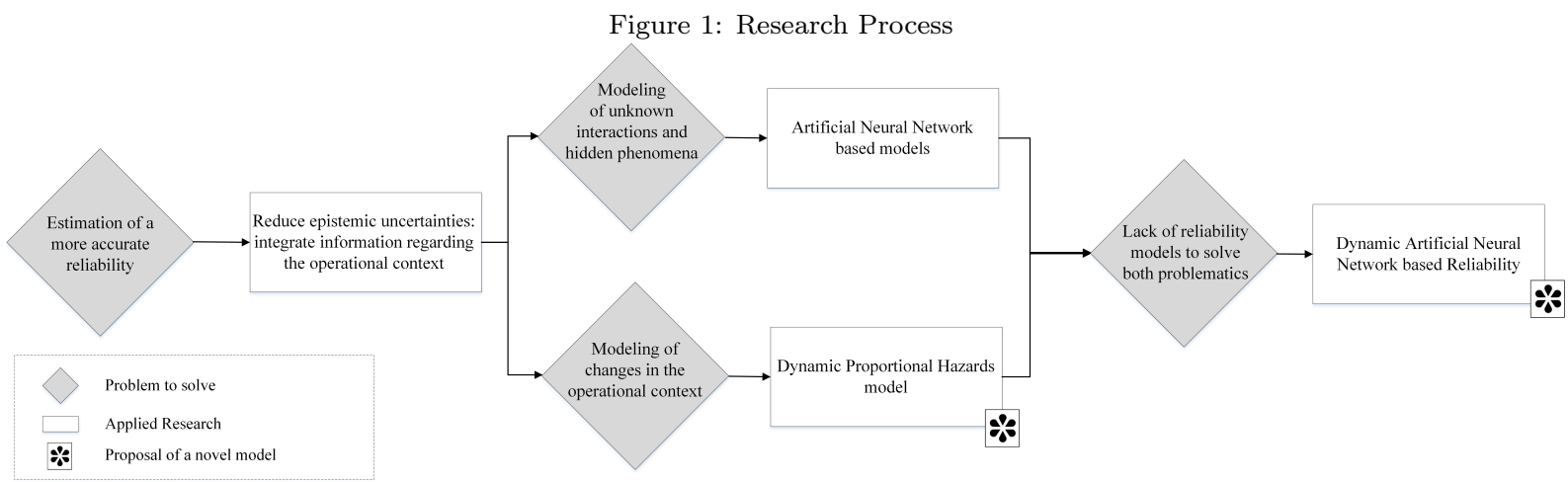

\subsection{Related works.}

The study of the affection that the operational environment has on asset management and reliability engineering aspects has received considerable attention lately. More specifically, interesting contributions regarding assets reliability can be found, for example in Okaro and Tao [13] the reliability of a subsea compression system is analyzed under operational covariate stresses, in Peng et al. [14] an approach taking into account dynamic conditions is proposed, for cable failure the operational context is explicitly modeled in Tang et al. [15] and also for traction transformers in Lin et al. [16]. However, reliability assessment is not the only aspect of asset management addressed in the literature with the affection of operational context to failures as a common underlying cause. For instance, spare parts estimation depending on the operational environment is studied in [17-19], availability assessment considering different weather conditions in [20] and maintainability analysis with context variables in [21, 22].

In the related literature the authors have gone through, the Proportional Hazards Model (PHM) is a recurrent model utilized for analyzing the effects of different variables of the operational context, therefore this model plays a key role as a starting point in the research. It was first introduced by Cox in 1972 [23] (it is also known as Cox model) and it was proposed for reliability studies by the author 
himself and by other researchers $[24,25]$. An early literature review of the first attempts of using the 75 model, along with a case study exemplifying its proper use, can be seen in [26]; through the paper, the work of Bendell facilitated the posterior applications of the model in reliability engineering. Afterward, several applications of the model can be found in many different industries and systems. The model have been proposed for the rail industry to predict rolling stock reliability, for instance in the door system [27] or the Heating Ventilating and Air Conditioning (HVAC) system [11]. It is also proposed for the so wind energy sector, to model failure rate in wind turbines [28] and to assess the turbines stress condition [29]. The research in the cutting tool industry also provides interesting applications of the model [30, 31]. And among others, the model have also been proposed for bearings reliability prediction [32], for the machinery in the processing industry [33], for electronic components [34,35] and for piping infrastructure [36].

Another recurrent approach found in the literature for integrating epistemic information regarding the operational context of the assets is the application of Artificial Neural Networks (ANN). As stated by Xu [37], the attractiveness of neural networks is mainly due to the fact that no 'a priori' assumption regarding the models is needed, and they have the advantage of representing complex nonlinear relationships as Marugán et al. [38] write. To date, several studies have begun to investigate the application of ANN for reliability problems, e.g.: in Al-Garni et al. [39] they are compared with the Weibull regression model, in Fink et al. [40] they are proposed to approach reliability prediction problems as time series, Beg et al. [41] present a comparison of several ANN-based models at estimating the probability of failure and Santosh et al. [42] propose an interesting approach for time to failure estimation combining Weibull regression models with ANNs.

In particular, the mentioned work of Santosh et al. [42] is a demonstration of the benefits of combining statistical models and ANN methods. Consistent with this approach it is important to emphasize the work of Faraggi and Simon [43] whose proposed model combines both of the previous approaches, ANNs architecture and the PHM. This ANN extension of the Cox model was conceived for studying rightcensored survival data [43] in the field of statistical medicine where most of its applications reside (see for instance $[44,45])$.

However this is not the only model in the literature that brings together both techniques; the work of Biganzoli et al. [46] focus on partial logistic models with ANN and feed forward ANN for modeling the hazard function, in Ripley et al. [47] seven survival models based on neural networks (both continuous and discrete time models) are described and Mazini et al. [48] proposes the use of neural networks to create a health condition model for wind turbines. As previously stated, the applications of these models remain almost exclusively in the discipline of medical statistics; nonetheless, there are already in the literature some attempts to extrapolate them to reliability engineering $[49,50]$.

When reliability engineering is considered within the organizations, it is related with maintenance management and therefore, with maintenance activities [51]. According to Crespo [52], the maintenance activities can either be classified as corrective maintenance, performed after the failure has happened; or preventive maintenance, dispatched at predefined intervals or according to certain criteria previously established. Each type of actions have an associated cost, corrective cost (CC) and preventive cost (PC), being on a general basis the $\mathrm{CC}$ greater [53]. Generally both types of costs include direct costs associated to manpower, replacement parts and materials, however the corrective costs not only is unplanned but also include indirect costs associated to penalization in terms of operation losses, impact on quality, environment or security among others [52]. In order to optimize the costs associated to maintenance activities, reliability modeling has been proposed as a useful tool [54]. In the literature can be found recent works that propose launching preventive maintenance activities according to a defined reliability threshold [55-57] being this the scenario intended for the later proposed model.

\subsection{Scientific contribution}

Based on the motivation stated in Subsection 1.1 and the related works presented in Subsection 1.2 , the research conducted by the authors has led to the development of a novel model for reliability estimation. As it is represented in figure 1, the main contribution of this paper is the proposal of a Dynamic Artificial Neural Network-based Reliability model which is a result of combining the concepts proposed for the ANN-based models and the Dynamic PHM, being the later a novel proposal in this paper as well.

The value proposition of the model is the improvement of reliability estimations, these better estimations rendered by the proposed model are mainly due to directly addressing three simplifications that other models have not dealt with altogether: 
A. The Dynamic ANN-based model not only takes into account the reliability driver but the operational context information as well. By integrating this information the model is built-up upon more complete knowledge of failure mechanisms, therefore, the epistemic uncertainty associated with the model is reduced.

B. The dynamic capabilities of the model directly address the reality of assets performing in changing operating contexts. The novel model here proposed has the capability of taking into account changes in the operational context and the length in the time of those changes, thus the model enhances reliability estimations in production peaks, seasonality, and other similar working environments with high variability in its operational conditions.

C. The proposed model brings in the advantages of integrating ANN. The ANN methods allow modeling unknown interactions between the different variables of the operational environment, without having to previously define them, as well as hidden phenomena which may be triggering or influencing the failure modes.

One important contribution of the research process is the proposal of the Dynamic PHM, which deals with items A and B. However, the apex of the scientific contribution of the research here presented is in the Dynamic ANN-based reliability model, which has the potential of addressing the three aforementioned key points into a single model.

\subsection{Overview}

The remainder of the paper consists of three sections, the following section presents the development of the Dynamic ANN-based model, then the model is tested on a case study in section 3 and finally the results and conclusions are discussed in the last section.

The development of the model in section 2 has followed the same research process previously presented in figure 1, and therefore the section is structured in a similar way. First, in order to set the foundations, the main introduction to PHM is presented, then in the following subsections 2.2 and 2.3 individual solutions for the top line and the bottom line of the applied research progress in figure 1 are respectively developed. Special emphasis is made on subsection 2.3 due to the novelty of the proposal stated in the previous subsection 1.3. And finally subsection 2.4 presents the main contribution of the paper, the Dynamic Artificail Neural Network-based reliability model which is conceived, and thus presented, as a combination of the previous models.

After laying down the theoretical dimensions of the model, the main topic covered in section 3 is the performance of the model in a case study as an illustrative example of its use and potential. The section consist of four subsections: in the first one the database and the case study are presented, then the optimization of the proposed model for the case study is explained, then the performance of the model is tested in terms of reliability estimations in the third and lastly cost-performance metrics are analyzed in the fourth subsection.

Finally, section 4, ties together up the various conclusions withdrawn during the research process, the model development and its application to the case study. Also some research lines worth of further investigation are presented here.

\section{Proposed model}

As previously stated, the proposed model takes as starting point the PHM and the concept of partial likelihood that Cox proposed along with it [23]. It is the concept of partial likelihood the one that will enable to integrate ANN methods with the PHM and therefore the section development follows this logic; starting by introducing the PHM with the partial likelihood, continuing with the ANN-based PHM, then the Dynamic extension of the Weibull PHM and finally the Dynamic ANN-based reliability model.

\subsection{Weibull Proportional Hazards Model}

The most common description of the PHM is the definition of the hazard rate provided by equation 1. In equation 1, the failure rate of a system is expressed as the product of a baseline hazard rate $h_{0}(t)$, as a function of the operating time of the asset $t$; and a term incorporating the operational context variables in an exponential form in which each one of the covariates $\mathbf{X}=\left(X_{1}, \ldots, X_{k}\right)$ is multiplied by a parameter $\boldsymbol{\beta}=\left(\beta_{1}, \ldots, \beta_{k}\right)$ describing its effect.

$$
h(t, \mathbf{X})=h_{0}(t) \exp \left(\sum_{j=1}^{k} \beta_{j} X_{j}\right)
$$


Table 1: Nomenclature definition

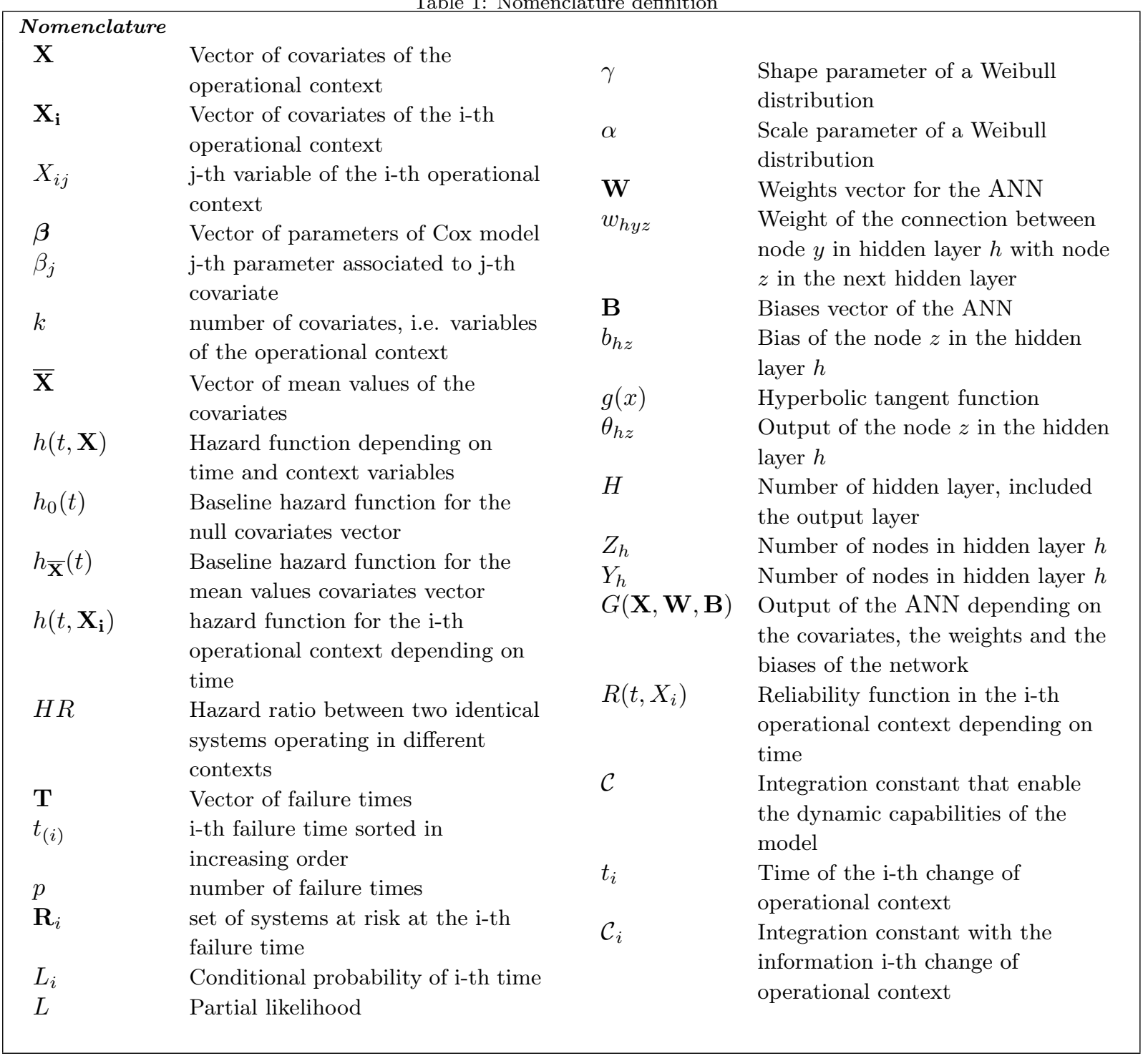

The baseline hazard function, $h_{0}(t)$, represents the hazard of a system operating in a context described by the null vectors of variables, $\mathbf{X}=\mathbf{0} \in \mathbb{R}^{k}$, and it can be parametric following certain distribution or of an unspecified form; and the operational context variables can be either a naturally variable or an indicator variable.

Given two identical systems operating in two different operational contexts, that would be $\mathbf{X}=$ $\left(X_{1}, \ldots, X_{k}\right)$ and $\mathbf{X}^{\prime}=\left(X_{1}^{\prime}, \ldots, X_{k}^{\prime}\right)$, being $\mathbf{X}^{\prime}$ the one with a higher risk; it is defined the Hazard Ratio (HR) between the two of them as:

$H R=\frac{h\left(t, \mathbf{X}^{\prime}\right)}{h(t, \mathbf{X})}=\frac{h_{0}(t) \exp \left(\sum_{j=1}^{k} \beta_{j} X_{j}^{\prime}\right)}{h_{0}(t) \exp \left(\sum_{j=1}^{k} \beta_{j} X_{j}\right)}=\exp \left(\sum_{j=1}^{k} \beta_{j}\left(X_{j}^{\prime}-X_{j}\right)\right)$

It can be observed from equation 2 that $\mathrm{HR}$ is independent of the system operating time. Thus, considering a special case of the HR in which the comparison is between a system operating in an environment where the covariates vector take mean values $\mathbf{X}=\left(\bar{X}_{1}, \ldots, \bar{X}_{k}\right)$ and the same system operating in a context $\mathbf{X}_{\mathbf{i}}=\left(X_{i 1}, \ldots, X_{i k}\right)$, the hazard function of the system operating in $\mathbf{X}_{\mathbf{i}}$ would be expressed by equation 3 , where the hazard function is decomposed into a baseline hazard function in the mean values of the covariates $\left(h_{\overline{\mathbf{x}}}(t)\right)$, and into the exponential part where the deviations from the mean value of each 
covariate are taken into account, instead of the covariates values themselves.

$h\left(t, \mathbf{X}_{\mathbf{i}}\right)=h_{\overline{\mathbf{X}}}(t) \exp \left(\sum_{j=1}^{k} \beta_{j}\left(X_{i j}-\bar{X}_{j}\right)\right)$

One of the main advantages of the Cox model is that the estimation of the parameters in the ex-

195 possible thanks to the concept of partial likelihood, it is defined as the product over every failure time of the conditional probability of failure of the system which actually failed at $t_{(i)}$. That is for a set of increasing ordered failure times $\mathbf{T}=\left(t_{(1)}, \ldots, t_{(i)}, \ldots, t_{(p)}\right)$, let $\mathbf{R}_{i}$ be the set of systems at risk at the time $t_{(i)}$, i.e. subjects which have not failed nor been censored before $t_{(i)}$, then the conditional probability is 200

$L_{i}=\frac{h\left(t_{i}, \mathbf{X}_{\mathbf{i}}\right)}{\sum_{l \in \mathbf{R}_{i}} h\left(t_{i}, \mathbf{X}_{\mathbf{l}}\right)}=\frac{\exp \left(\sum_{j=1}^{k} \beta_{j}\left(X_{i j}-\bar{X}_{j}\right)\right)}{\sum_{l \in \mathbf{R}_{i}} \exp \left(\sum_{j=1}^{k} \beta_{j}\left(X_{l j}-\bar{X}_{j}\right)\right)}$

$L=\prod_{i=1}^{p} L_{i}$

The estimation of the parameters is performed by maximizing the partial likelihood in equation 5 , traditionally by Newton-Raphson iterations; as it can be observed the effects of the variables of the operational context are estimated without making any assumptions regarding the baseline hazard function. However, it is interesting for the scope of the paper to introduce the full parametric version of model in which the baseline hazard function in the mean values of the covariates is fitted to follow a two-parameter Weibull distribution. This alternative of the Cox model is represented in equation 6, where $\gamma$ is the shape parameter and $\alpha$ is the scale parameter, and it is known as Weibull PHM.

$h\left(t, \mathbf{X}_{\mathbf{i}}\right)=\frac{\gamma}{\alpha} \cdot\left(\frac{t}{\alpha}\right)^{\gamma-1} \cdot \exp \left(\sum_{j=1}^{k} \beta_{j}\left(X_{i j}-\bar{X}_{j}\right)\right)$

\subsection{Neural-Network based reliability model}

In the previous section, it can be seen that the combination of the operational context variables is linear in the most simple form of the Weibull PHM, or "a priori" defined in other variations. Thus, this feature limits the suitability of the model for modeling unknown interactions or hidden phenomena and it is in this issue where the architecture of the ANNs provides value.

The proposed model is based on feed-forward neural networks with only one output node, and the operational context variables, $\mathbf{X}=\left(X_{1}, \ldots, X_{k}\right)$, will form the input nodes, a graphical representation can

215 be seen in figure 2 . The inputs and the output are connected through hidden layers with different nodes number. Every connection between nodes is associated with a weight parameter, all of them represented by the vector $\mathbf{W}$ where each position is described by $w_{h y z}$, being $h$ the origin layer (starting in 0 for the input layer and ending in $\mathrm{H}$ for the output layer), $y$ represent the node in the origin layer and $z$ the destination node in the next layer. Every node in the hidden layers is also associated with a bias term

all of them represented by vector $\mathbf{B}$ where each position is described by $b_{h z}$ being $h$ the hidden layer and $z$ the node in the corresponding hidden layer. In every neuron, also known as computational unit, an activation function is applied to the input received, in the proposed model the activation function $g(x)$ is the hyperbolic tangent, which is described in equation 7 and have proofed to train faster than the sigmoid activation [44].

$g(x)=\frac{\exp (x)-\exp (-x)}{\exp (x)+\exp (-x)}$ 


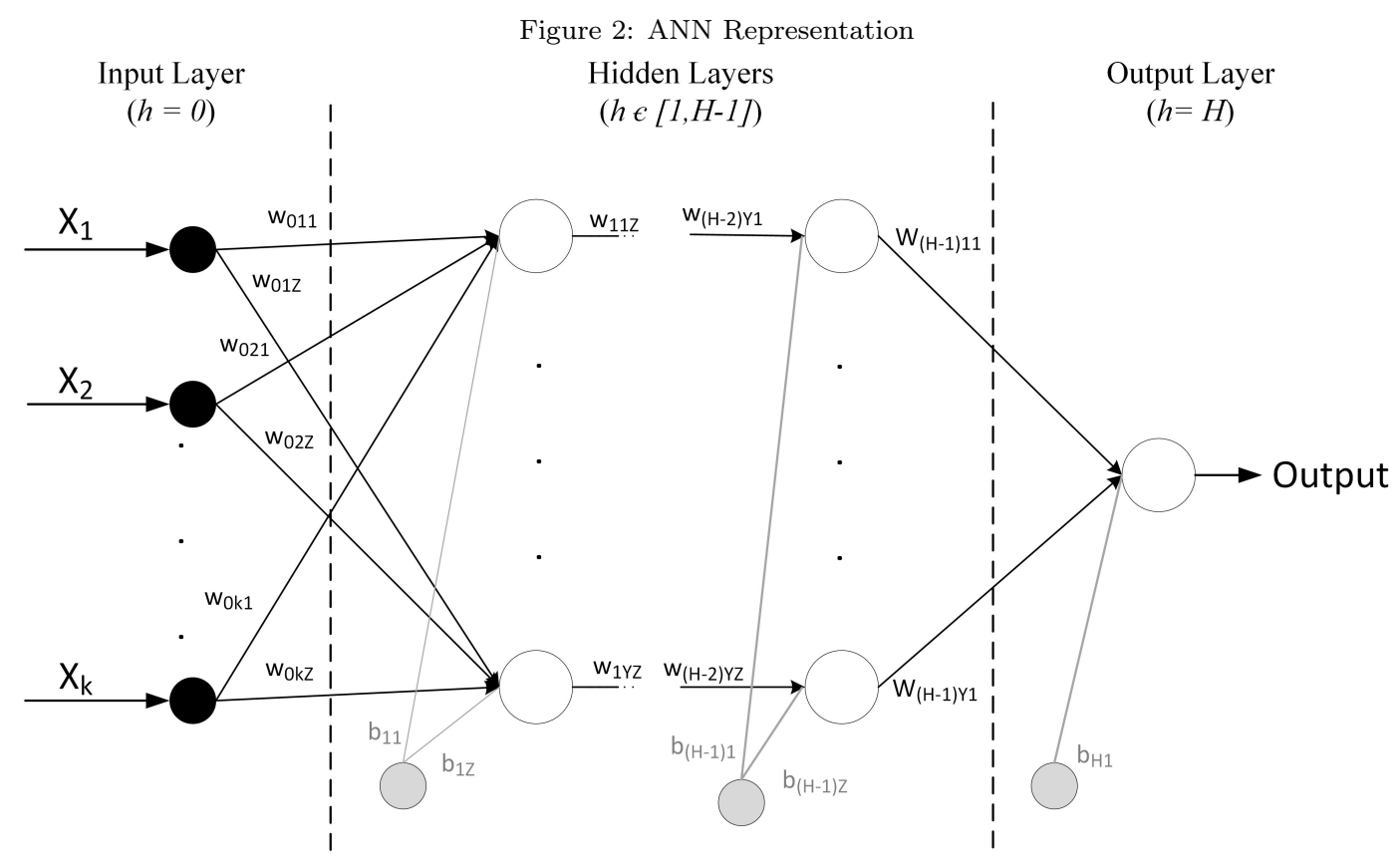

Table 2: ANN equations

\begin{tabular}{lcc}
\hline Layer & $\begin{array}{c}\text { Output of the nodes in the } \\
\text { layer }\end{array}$ & Domain \\
\hline First hidden Layer $(\mathrm{h}=1)$ & $\theta_{1 z}=g\left(\sum_{y=1}^{k} w_{0 y z} X_{y}+b_{1 z}\right)$ & $\forall z \epsilon\left[1, Z_{h=1}\right]$ \\
Next hidden Layers & $g\left(\sum_{y=1}^{Y_{h-1}} w_{(h-1) y z} \theta_{(h-1) y}+b_{h z}\right)$ & $\forall h \epsilon[2,(H-1)] \forall z \epsilon\left[1, Z_{h}\right]$ \\
Output layer $(\mathrm{h}=\mathrm{H})$ & $g\left(\sum_{y=1}^{Y_{H-1}} w_{(H-1) y 1} \theta_{(H-1) y}+b_{H 1}\right)$ & \\
\end{tabular}

$\mathrm{g}(\mathrm{x})$ is the previously defined hyperbolic tangent function

The equations that govern the ANN described in the paragraph above and represented in figure 2 can be seen in Table 2, the equations have been organized according to the different layers and the calculations that involve every one of them. The term $\theta_{h z}$ refers to the output of the neuron $z$ of the hidden layer $h$.

Considering the application of the equations on Table 2 , it is possible to define the network as a non-linear complex function depending on the covariates vector $\mathbf{X}=\left(X_{1}, \ldots, X_{k}\right)$, the weights of the connections between the nodes $\mathbf{W}$, and the bias terms $\mathbf{B}$. This function is denoted by $G(\mathbf{X}, \mathbf{W}, \mathbf{B})$ and the Neural-Network based reliability model propose replacing the linear $\sum_{j=1}^{k} \beta_{j} X_{i j}$ in the Cox model by the output of the neural network function denoted by $G(\mathbf{X}, \mathbf{W}, \mathbf{B})$. This proposal is described by equation 8 , and with this model the partial likelihood function $(L)$ to maximize in order to estimate the neural network parameters(weights and bias terms) would be equation 9 .

$h(t, \mathbf{X})=h_{0}(t) \exp (G(\mathbf{X}, \mathbf{W}, \mathbf{B}))$

$L=\prod_{i=1}^{p} \frac{\exp \left(G\left(\mathbf{X}_{\mathbf{i}}, \mathbf{W}, \mathbf{B}\right)\right)}{\sum_{l \epsilon \mathbf{R}_{i}} \exp \left(G\left(\mathbf{X}_{l}, \mathbf{W}, \mathbf{B}\right)\right)}$ 
It is important to notice that in this model the parameters of the neural network are not obtained by training it but by maximizing the partial likelihood function, expressed in equation 9. Nonetheless, depending on the chosen architecture of the ANN the maximization of equation 9 may result in a very complex and computationally intensive problem; thus a genetic algorithm is proposed for maximizing the partial likelihood.

\subsection{Dynamic Weibull-Proportional Hazards Model}

Taking as a starting point the hazard rate described by the Weibull PHM in equation 6, the reliability derived from that particular expression of the failure rate of a system can be calculated as follows:

$$
\begin{aligned}
& R\left(t, X_{i}\right)=\exp \left(-\int h\left(t, X_{i}\right) d t\right) \\
& R\left(t, X_{i}\right)=\exp \left(-\int \frac{\gamma}{\alpha}\left(\frac{t}{\alpha}\right)^{\gamma-1} \exp \left(\sum_{j=1}^{k} \beta\left(X_{i j}-\overline{X_{j}}\right)\right) d t\right) \\
& R\left(t, X_{i}\right)=\exp \left(-\left(\frac{t}{\alpha}\right)^{\gamma} \exp \left(\sum_{j=1}^{k} \beta\left(X_{i j}-\overline{X_{j}}\right)\right)+\mathscr{C}\right)
\end{aligned}
$$

In the general reliability function of equation 12 the variables of the operational context do not depend on time, the shape and scale parameters are considered to be constants inherent to the technical characteristics of the system and the parameter $\mathscr{C}$ is the integration constant.

When the asset starts to operate in a context A, i.e. $\mathbf{X}_{\mathbf{A}}=\left(X_{A 1}, \ldots, X_{A k}\right)$, the reliability of the asset is $100 \%$ and therefore $R\left(t=0, X=X_{A}\right)=1$, by solving the equation for the integration constant $\mathscr{C}_{A}$ it can be seen that its value equals 0 for any value of $\mathbf{X}_{\mathbf{A}}=\left(X_{A 1}, \ldots, X_{A k}\right)$. However after operating in context $\mathrm{A}$ during certain time $t=t_{1}$ the operational context changes to $\mathrm{B}$ which is described by $\mathbf{X}_{\mathbf{B}}=\left(X_{B 1}, \ldots, X_{B k}\right)$, and the system reliability is going to be described now by equation 13 .

$R\left(t, X_{B}\right)=\exp \left(-\left(\frac{t}{\alpha}\right)^{\gamma} \exp \left(\sum_{j=1}^{k} \beta\left(X_{B j}-\overline{X_{j}}\right)\right)+\mathscr{C}_{B}\right)$

To obtain the unknown value of $\mathscr{C}_{B}$ it is necessary to solve the equation $R\left(t_{1}, X_{A}\right)=R\left(t_{1}, X_{B}\right)$, and the same reasoning will be followed to calculate system reliability if the asset changes in $t=t_{2}$ from operational context B to operational context C. Generalizing this logical process leads to the Equations 14 and 15.

$R\left(t, X_{i}\right)=\exp \left(-\left(\frac{t}{\alpha}\right)^{\gamma} \exp \left(\sum_{j=1}^{k} \beta\left(X_{i j}-\overline{X_{j}}\right)\right)+\mathscr{C}_{i}\right)$

$\mathcal{C}_{i}=\left\{\begin{array}{cc}0 & \forall i=0 \\ \left(\frac{t_{i}}{\alpha}\right)^{\gamma}\left(\exp \left(\sum_{j=1}^{k} \beta\left(X_{i j}-\overline{X_{j}}\right)\right)-\exp \left(\sum_{j=1}^{k} \beta\left(X_{(i-1) j}-\overline{X_{j}}\right)\right)\right)+\mathcal{C}_{i-1} & \forall i \neq 0\end{array}\right.$

The $\mathscr{C}_{i}$ parameter is the term in which all the information regarding the operational context changes is stored, hence its recursiveness towards its value in the previous operational context. It acts as an indicator of the state of the system in terms of reliability, linking the new reliability curve to be followed with the previous evolution of the asset. In this parameter the sub-index $i$ is for the operational context changes starting in the first context with $i=0$. Taking a closer look at the different elements of the expression of the $\mathscr{C}_{i} \forall i \neq 0$ it is possible to explain its meaning by dividing it into three terms: 
- $\left(\frac{t_{i}}{\alpha}\right)^{\gamma}$. The changes in the operational context do not affect the asset in the same way during the whole span of its operating time. The impact of the changes will depend on the time at which they happen and also on the characteristic of the asset. This term integrates the aforementioned details; the time at which the change happens is explicitly represented $\left(t_{i}\right)$ and asset characteristics are included in the characterization of the reliability curve, by the shape $(\beta)$ and scale $(\alpha)$ parameters.

- $\left(\exp \left(\sum_{j=1}^{k} \beta\left(X_{i j}-\overline{X_{j}}\right)\right)-\exp \left(\sum_{j=1}^{k} \beta\left(X_{(i-1) j}-\overline{X_{j}}\right)\right)\right)$. This term integrates in the constant information regarding how different is the new operational context from the previous, the bigger the differences the more effect will have the constant on the new reliability curve which the system will follow.

- $\mathcal{C}_{i-1}$. The system state will also depend on previous changes it had gone through and therefore, it is this term the one that integrates it by gathering the information of the constant from the previous operational context change.

As the changes in the operational context are not specified but defined according to time intervals, the model allows for a dynamic calculation of the reliability. Besides, the length of the time intervals are not

275 defined, thus, they can be of any length and they do not have to be of the same duration; the longer the time interval the more aggregated the information and therefore the less accurate the estimations, but still better than not considering any change in the operational conditions.

\subsection{Dynamic Artificial Neural Network-based reliability model}

By now the Neural Network based reliability has been introduced in subsection 2.2 and its main contribution is the possibility of modeling unknown interactions among the variables and hidden phenomena. Also the subsection 2.3 the Dynamic Weibul PHM that allows calculating system reliability taking into account changes in the operational context and their time span. Here in this subsection, the combination of both models is developed in order to achieve a model which comprehensively gathers the benefits from both approaches.

Consider the hazard proposed by the Neural Network based reliability model, however, the baseline hazard is going to be adjusted to the mean values of the operational context, equation 16. From there, the calculation of the reliability follows the same logical process as in the Dynamic Weibull PHM. As it can be observed, the output of the Artificial Neural Net does not depend on time, thus, for integration purposes it is considered a constant. Then the following equations are derived through the same calculations as in the Dynamic Weibull Proportional Hazards section, the resulting reliability function is the one described in equation 18 and the value of the $\mathscr{C}_{i}$ term is described in equation 19.

$$
h(t, \mathbf{X})=h_{\overline{\mathbf{X}}}(t) \exp (G((\mathbf{X}-\overline{\mathbf{X}}), \mathbf{W}, \mathbf{B}))
$$

$R\left(t, X_{i}\right)=\exp \left(-\int \frac{\gamma}{\alpha}\left(\frac{t}{\alpha}\right)^{\gamma-1} \exp (G((\mathbf{X}-\overline{\mathbf{X}}), \mathbf{W}, \mathbf{B})) d t\right)$

$R\left(t, X_{i}\right)=\exp \left(-\left(\frac{t}{\alpha}\right)^{\gamma} \exp \left(G\left(\left(\mathbf{X}_{\mathbf{i}}-\overline{\mathbf{X}}\right), \mathbf{W}, \mathbf{B}\right)\right)+\mathscr{C}_{i}\right)$

$\mathcal{C}_{i}=\left\{\begin{array}{cc}0 & \forall i=0 \\ \left(\frac{t_{i}}{\alpha}\right)^{\gamma}\left(\exp \left(G\left(\left(\mathbf{X}_{\mathbf{i}}-\overline{\mathbf{X}}\right), \mathbf{W}, \mathbf{B}\right)\right)-\exp \left(G\left(\left(\mathbf{X}_{\mathbf{i}-\mathbf{1}}-\overline{\mathbf{X}}\right), \mathbf{W}, \mathbf{B}\right)\right)\right)+\mathcal{C}_{i-1} & \forall i \neq 0\end{array}\right.$

With the proposal of this new model, it is possible not only to integrate operational context variables but to model the interactions among them that may trigger or condition failures. Besides it is possible to take into account the operational changes in terms of duration, differences in the operational environment and system age at which the changes happen. In the following section, a case study is developed exemplifying the application of the model and its benefits. 


\section{Model performance. Case study}

A case study approach was adopted to provide evidence on the value added by the proposed model, to such aim it has been tested against two models of wide application. The first compared model is the traditional two-parameter Weibull distribution, which estimates the reliability of the assets based just on the operating time and therefore it does not integrate information regarding the operational context. The other model included in the comparison is the Weibull-PHM, i.e. the one in equation 6 , which integrates information regarding the operational context but in a linear combination of the variables, thus it does not consider interactions among the operational context variables nor does it have dynamic capabilities.

\subsection{Data base and case study}

The case study is based on a database of aircraft engines failures with their corresponding operating conditions. More precisely, the database contains the random failures of over 1000 assets with their daily operational context characterized by 17 variables. It also contains right-censored data of those assets which have not failed and are associated to certain operational conditions as well.

In order to fit the model and test its performance, the data base has been split into a train dataset and a test data-set. The train data set contains 355 failure events and 40 right-censored data and the test data-set contains 354 failures and 704 censored data. Both fault and censored data have their corresponding daily operating conditions associated with them. The train data-set is intended for the model fitting, also for the Weibull and the Weibull-PHM models fitting; and the test data set is used for comparing the performance of the three models estimating the reliability.

\subsection{Model selection and fitting}

Prior to testing and comparing the performance of the models, the architecture of the ANN in the exponential part of the Dyanmic ANN-based reliability, equation 18, should be selected. To this aim several configurations of hidden layers and nodes have been proposed and optimized for the data-set; the the followed to determine which one to choose has been the maximization of the vained for the partial likelihood. The different architectures have been optimized through a genetic algorithm, the obtained values for the partial likelihood as well as the number of parameters to optimize in every option is shown in Table 3. It can be observed that the one with the best result is the 3-1 architecture consisting of two hidden layers with 3 and 1 hidden nodes correspondingly.

Having selected the best architecture of the ANN with its corresponding optimized parameters, the next step is to fit the baseline hazard to follow a two-parameter Weibull distribution. For the selected architecture of ANN, the shape and scale parameters of the baseline hazard function have a value of 4.2 and 206.7 correspondingly.

In short, the Dynamic ANN-based reliability model consists of a baseline hazard function characterized by a shape and scale parameter and an exponential part with the ANN architecture consisting of two hidden layers which contain 3 neurons the first and 1 neuron the second being the hyperbolic tangent the activation function.

Table 3: ANN architectures performance

\begin{tabular}{cccc}
\hline $\begin{array}{c}\text { Number of hidden } \\
\text { layers }\end{array}$ & Architecture & $\begin{array}{c}\text { Number of parameters } \\
\text { to optimize }^{1}\end{array}$ & $\begin{array}{c}\text { Partial Likelihood } \\
\text { Value }\end{array}$ \\
\hline \hline $\mathrm{H}=1$ & 2 & $5+2 k$ & -1745.482 \\
$\mathrm{H}=1$ & 3 & $7+3 k$ & -1743.431 \\
$\mathrm{H}=1$ & 4 & $9+3 k$ & -1746.126 \\
$\mathrm{H}=2$ & $2-1$ & $7+2 k$ & -1743.576 \\
$\mathrm{H}=2$ & $2-2$ & $11+2 k$ & -1747.799 \\
$\mathrm{H}=2$ & $3-1$ & $9+3 k$ & -1738.314 \\
$\mathrm{H}=2$ & $3-2$ & $14+3 k$ & -1747.492 \\
$\mathrm{H}=2$ & $3-3$ & $19+3 k$ & -1743.467 \\
$\mathrm{H}=2$ & $4-1$ & $11+4 k$ & -1748.591 \\
$\mathrm{H}=2$ & $4-2$ & $17+4 k$ & -1741.402 \\
$\mathrm{H}=2$ & $4-3$ & $23+4 k$ & -1745.660 \\
$\mathrm{H}=2$ & $4-4$ & $29+4 k$ & -1747.065 \\
\hline
\end{tabular}

1-The number of parameters to optimize depends on $k$, it refers to the number of input variables. 


\subsection{Test and validation of the model} to the widely utilized Weibull and Weibull-PHM reliability models to see if it provides any improvement in reliability estimations. To such aim, the three models are tested by estimating the reliability of the assets in the test data-set. The traditional Weibull model estimates the reliability of the assets according to their operation times when they failed or were censored. The Weibull-PHM performs the estimations ANN-based reliability model estimates the assets probability of failure by considering operational time, the daily changes in operational conditions (given its dynamic capability) and the possible interactions among the variables.

As the proposed reliability model aims at improving the efficacy and accuracy of maintenance stratees, several maintenance thresholds have been established representing different scenarios in which the preventive maintenance actions are triggered when the reliability of the assets reach the threshold. Therefore, according to the reliability estimated by the models and the maintenance threshold, the assets in the test data-set may be maintained, effectively in the failure cases or unnecessarily in the censored cases (non failure). In consonance with this logic, a new concept of reliability-based confusion matrix has been defined, its schematic view can be seen in figure 3 and it is based in the following terms:

- True positive (TP). This measure represents the failures which would have been avoided due to the preventive action, given certain reliability threshold as criterion to launch preventive maintenance on assets. As the models estimate a different reliability for the assets, the choice of the reliability model directly conditions assumed failures. This measure is associated with the cost of preventive maintenance, i.e. the cost incurred for every asset maintained before the failure (PC). As the failure would had occurred if the asset had not been maintained, this cost is called in the paper Effective Preventive Cost (EPC).

- False positive (FP). Some assets may reach the reliability threshold for maintenance in the different estimates of the three models, yet they are not failure data but censored data in the test data-set. It means that the preventive maintenance performed to such assets would have been unnecessary actions and hence unnecessary PC. Therefore the FPs for every model are associated with Over-maintenance Preventive Cost (OPC).

- False negative (FN). In other cases failing assets may not reach the reliability threshold and yet they are failure data. This measure is intended to represent the failures assumed by choosing certain maintenance threshold and will depend on whether the models estimates reach the threshold before the failure time or not. If they do not reach the threshold, it means the asset would not have been maintained and therefore corrective maintenance actions taken would have incurred in CC.

- True negatives (TN). The TN indicator represents all the censored assets which would not have been maintained because the reliability estimates do not reach certain maintenance threshold, therefore, not preventive nor corrective maintenance actions are taken on the assets. There is no cost associated to the TN since they are not failures and they are not subject to preventive maintenance actions.

The three models have estimated the reliability of assets in the test data-set at failure or censoring time. With the estimates, various confusion matrices have been calculated for different reliability thresholds, which range from a very risky maintenance policy (maintaining assets at reliability threshold of 10\%) to a highly conservative one (maintaining assets at reliability threshold of 90\%) with four intermediate positions. According to the thresholds and the reliability estimated by the models, the proportion of TPs, FPs, FNs and TNs is different for each one of the three models. The results yielded may be observed in the confusion matrices of figure 3 .

As shown, with respect to the traditional Weibull model, the Dynamic ANN-based model avoids more failures (TP) in every scenario reaching a peak of improvement of $446 \%$ when the threshold equals 0.1 . However, the proposed model also over-maintains more assets for every threshold with a peak of a $277 \%$ higher number of over-maintained assets with respect to the Weibull model in the 0.1 threshold as well, meaning that reliability estimations are in general lower for the Dynamic ANN-based model.

Comparing the Dynamic ANN-based reliability with the Weibull-PHM, it is important to notice that the most significant difference in the TPs is in the 0.1 maintenance threshold with a $65 \%$ more of avoided failures for Dynamic ANN-based model. Also in this comparison the Dynamic ANN-based reliabiliy 
over-maintain more assets reaching a $48 \%$ higher number of assets unnecessarily maintained in the 0.1 threshold.

It is significant that in most threshold the proposed model avoids more failures than assets overmaintains in comparison with the traditional Weibull reliability and the Weibull-PHM. This result further validates the advantages of the model, specially considering that the test data-set have a considerably higher proportion of non-failure data as described in subsection 3.1.

Figure 3: Confusion matrices for both models and several maintenance thresholds
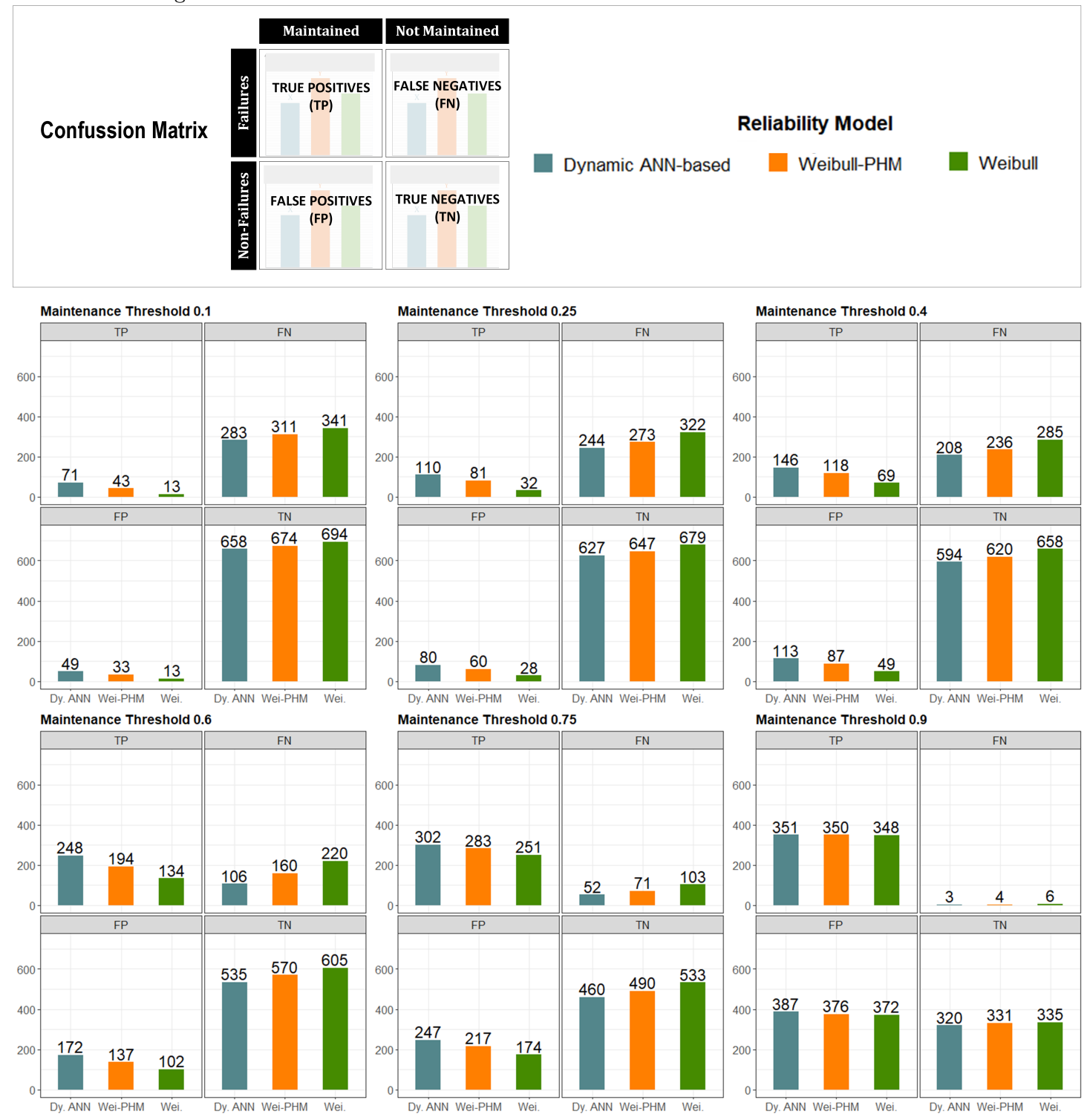

\subsection{Cost performance}

In the previous subsection it has been proved that the proposed model improves failure avoidance and to a lesser extent it incurs in undue maintenance. Nonetheless, the impact of both realities it is usually not of equal importance, the over preventive maintenance is preferable because the PC is usually much lower than CC and it can be planned as stated previously in subsection 1.2. Therefore, to further enrich the results analysis, cost-performance metrics have been analyzed. Following the concepts of the confusion matrix, the total cost of a maintenance policy based on reliability thresholds for the assets in test data-set can be decomposed as the sum of three different costs associated to TP, FP and FN; this total cost is expressed in equation 20 . 
It is possible to define the Maintenance Costs Ratio (MCR) as the quotient between CC and PC, see equation 21. Therefore the total cost of the maintenance policy for the assets in the test data-set can be expressed by equation 22 as well. This formulation of the total cost allows to represent in figure 4 three scenarios for different MCR. In the graphs is represented the cost of the maintenance policy for the models and every possible maintenance threshold. Besides, along with the costs, the savings derived from estimating the reliability with the Dynamic ANN-based model instead of the Weibull or Weibull-PHM models is depicted.

$M C R=\frac{C C}{P C}=\frac{C C}{O P C}=\frac{C C}{E P C}$

Total Cost $=(M C R \cdot F N+T P+F P) \cdot P C$

Figure 4: Marginal costs comparison and savings between models

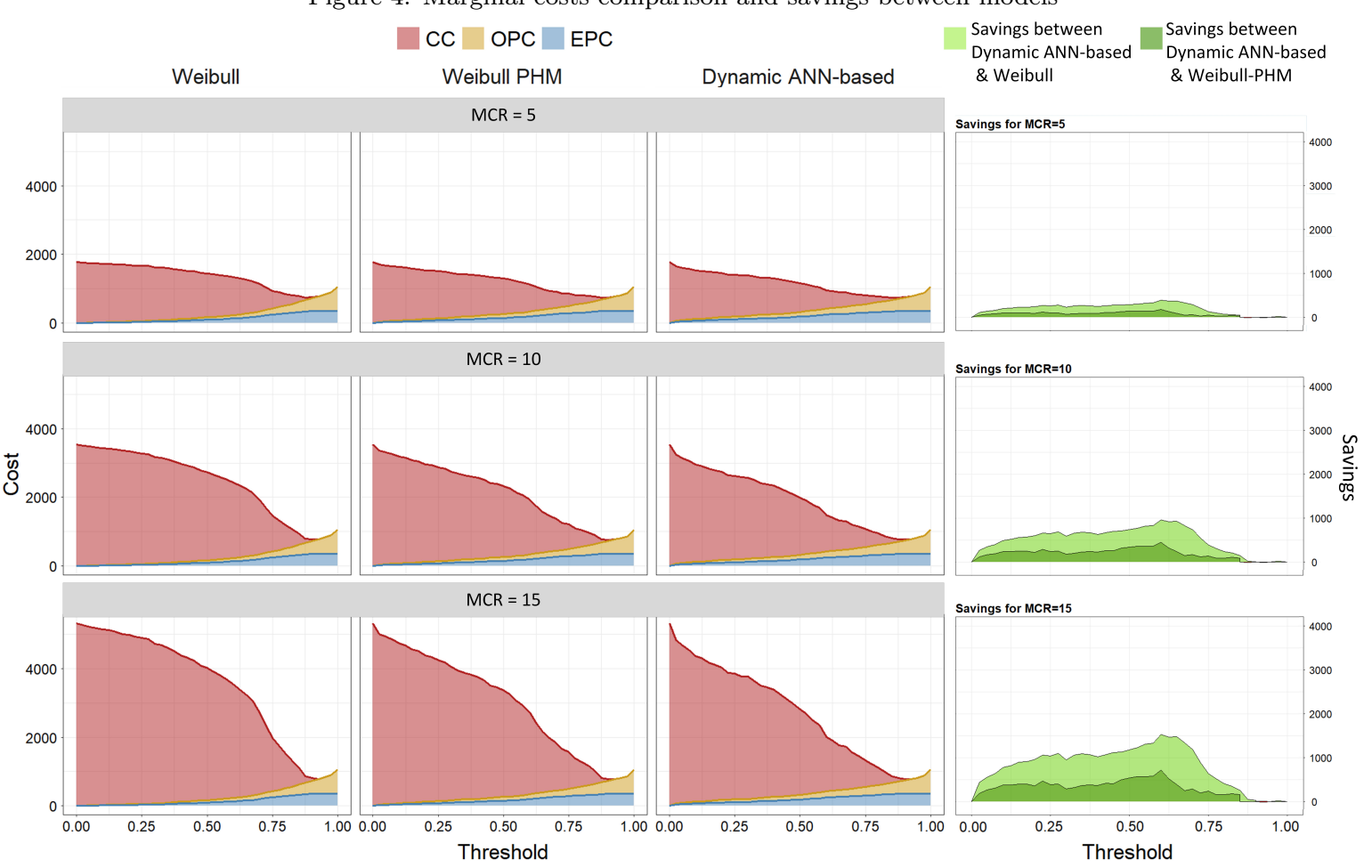

Three different scenarios according to the Maintenance Cost Ratio (MCR) are represented

The Cost and Savings, in the left and right vertical axis correspondingly, are expressed in Monetary Units (MU) per Cost of Preventive maintenance action $(P C)$, it is derived from the formulation of equation 22 .

In figure 4 it can be seen that for every threshold in each one of the three MCR scenarios represented, the model with the best cost-perfomance is the Dynamic ANN-based Reliability; this is also observable in the savings graph. The differences between the models mainly resides in the CC associated to each one of them. The differences yielded by the models are increasingly prominent as the Maintenance Cost Ratio (MCR) grows, i.e. the higher the $\mathrm{CC}$ with respect to the PC the better performs the proposed model and so does reflect the savings graphs.

It is interesting to remark that the more information the models integrate, the better their performance is. In the Weibull-PHM the operational context information is integrated in an aggregate way and thus it outperforms the traditional Weibull reliability model. In the Dynamic ANN-based model the operational context information is integrated daily considering interactions among the variables as well and it shows better performance than the Weibull-PHM. Herein the three models are compared for reliability estimates, they are of growing complexity but yield better estimations by integrating more information and hence reducing epistemic uncertainty. 
It is important to notice that the evolution of the $\mathrm{CC}$ for each one of the models is quite revealing, their comparison shows further evidence on the performance of the model. On the one hand, in the Dynamic ANN-based model and in the Weibull-PHM the evolution shows a nearly constant decrease in every MCR scenario, it suggests that the reliability estimations are accurate and that the failures responsible for the $\mathrm{CC}$ are the ones explicitly assumed by the chosen maintenance policy, i.e. the selected threshold. On the other hand, turning to the Weibull model and its CC, the slope intensifies in higher threshold values indicating that a considerable amount of failures avoided in such scenarios are due to the highly conservative maintenance strategy, instead of being attributed to the accuracy of the model.

\section{Conclusions}

The main objective of the research presented in this paper is to reduce the epistemic uncertainty inherent to current reliability estimations. The dynamic artificial neural network-based model presented integrates information regarding the operational context of the assets, hence, reducing to some extent the uncertainty since more information is translated into knowledge and explicitly considered in the model. Moreover, it deals with two common assumptions in many traditional proposals for reliability estimations that are "constant operational contexts" and "no interaction among operational variables" reducing to a further extent the uncertainty involved in the model estimations.

The dynamic capability of the model is characterized by the possibility of taking into account the heterogeneity in the span of the operational changes or the intervals in which the information has been recorded. Thus, it increases the capability of the model to adapt to different problematic scenarios in real industry practice. This information regarding operational context changes and its impact on the asset reliability is all comprehended in a single term, reducing the storage and computational capacity needed to collect and process that information by traditional techniques.

In the case study presented in this paper, the results show how the dynamic possibilities of the model along with the architecture of the artificial neural networks are able to better estimate assets reliability in order to enable a more accurate and therefore a better-customized maintenance policy. The proposed model implies a lower risk level in the maintenance management and its decision-making process, it is a step forward in the process of offering assets and its associated services with less uncertainty.

The research herein presented give rise to interesting questions whose answers remain open for future research lines. The benefits of considering the operational context is captured in the research, as well as the importance of dynamic considerations; however, by integrating the artificial neural network architecture it is more complicated to interpret the separate effect of each one of the context variables being this issue worth of further research and consideration. This is an important aspect to take into account if the operational context impact on assets reliability were to be address on early stages of the life cycle of the assets.

\section{Funding}

This research work was performed within both the context of SustainOwner ('Sustainable Design and Management of Industrial Assets through Total Value and Cost of Ownership'), a project sponsored by the EU Framework Programme Horizon 2020, MSCA-RISE-2014: Marie Sktodowska-Curie Research and Innovation Staff Exchange (RISE) (grant agreement number 645733 - Sustain-Owner - H2020-MSCARISE-2014) and the EmaitekPlus 2018-2019 Program of the Basque Government.

\section{References}

[1] E. Zio, Reliability engineering: Old problems and new challenges, Reliability Engineering \& System Safety 94 (2) (2009) 125 - 141. doi:https://doi.org/10.1016/j.ress.2008.06.002. URL http://www.sciencedirect.com/science/article/pii/S0951832008001749

[2] F. Chiacchio, D. D'Urso, G. Manno, L. Compagno, Stochastic hybrid automaton model of a multi-state system with aging: Reliability assessment and design consequences, Reliability Engineering \& System Safety 149 (2016) 1 - 13. doi:https://doi.org/10.1016/j.ress.2015.12.007. URL http://www.sciencedirect.com/science/article/pii/S0951832015003592

[3] R. Kang, Z. Qingyuan, Z. Zhiguo, Z. Enrico, L. Xiaoyang, Measuring reliability under epistemic uncertainty: Review on non-probabilistic reliability metrics, Chinese Journal of Aeronautics 29 (3) (2016) 571 - 579. doi:https://doi. org/10.1016/j.cja.2016.04.004. URL http://www.sciencedirect.com/science/article/pii/S1000936116300231

[4] W. Yao, X. Chen, Y. Huang, M. van Tooren, An enhanced unified uncertainty analysis approach based on first order reliability method with single-level optimization, Reliability Engineering \& System Safety 116 (2013) 28 - 37. doi:https://doi.org/10.1016/j.ress.2013.02.014.

URL http://www.sciencedirect.com/science/article/pii/S0951832013000495 
[5] S. Nannapaneni, S. Mahadevan, Reliability analysis under epistemic uncertainty, Reliability Engineering \& System Safety 155 (2016) 9 - 20. doi:https://doi.org/10.1016/j.ress.2016.06.005.

URL http://www.sciencedirect.com/science/article/pii/S0951832016301119

[6] J. Shortridge, T. Aven, S. Guikema, Risk assessment under deep uncertainty: A methodological comparison, Reliability Engineering \& System Safety 159 (2017) 12 - 23. doi:https://doi.org/10.1016/j.ress.2016.10.017. URL http://www.sciencedirect.com/science/article/pii/S095183201630713X

[7] T. Nilsen, T. Aven, Models and model uncertainty in the context of risk analysis, Reliability Engineering \& System Safety 79 (3) (2003) 309 - 317. doi:https://doi.org/10.1016/S0951-8320(02) 00239-9. URL http://www.sciencedirect.com/science/article/pii/S0951832002002399

[8] P. Weber, G. Medina-Oliva, C. Simon, B. Iung, Overview on bayesian networks applications for dependability, risk analysis and maintenance areas, Engineering Applications of Artificial Intelligence 25 (4) (2012) 671 - 682, special Section: Dependable System Modelling and Analysis. doi:https://doi.org/10.1016/j.engappai.2010.06.002. URL http://www.sciencedirect.com/science/article/pii/S095219761000117X

[9] D. Louit, R. Pascual, A. Jardine, A practical procedure for the selection of time-to-failure models based on the assessment of trends in maintenance data, Reliability Engineering \& System Safety 94 (10) (2009) 1618 - 1628. doi:https://doi.org/10.1016/j.ress.2009.04.001. URL http://www.sciencedirect.com/science/article/pii/S0951832009000891

[10] L. Yang, K. He, Y. Guo, Reliability analysis of a nonlinear rotor/stator contact system in the presence of aleatory and epistemic uncertainty, Journal of Mechanical Science and Technology 32 (9) (2018) 4089-4101. doi:10.1007/ s12206-018-0808-2.

URL https://doi.org/10.1007/s12206-018-0808-2

[11] J. Izquierdo, Assessing the impact of operational context variables on rolling stock reliability. a real case study, in: C. Press (Ed.), Safety and Reliability - Safe societies in a changing world, Proceedings of ESREL 2018, Taylor \& Francis Group, London, Trondheim, Norway, 2017, pp. 571-578. URL https://www.taylorfrancis.com/books/9781351174657

[12] J. Brings, A. Salmon, S. Saritas, Context uncertainty in requirements engineering definition of a search strategy for a systematic review and preliminary result., in: REFSQ Workshops, 2015, pp. 171-178. URL http://ceur-ws.org/Vol-1342/

[13] I. A. Okaro, L. Tao, Reliability analysis and optimisation of subsea compression system facing operational covariate stresses, Reliability Engineering \& System Safety 156 (2016) 159 - 174. doi:https://doi.org/10.1016/j.ress.2016. 07.018.

URL http://www.sciencedirect.com/science/article/pii/S0951832016302903

[14] W. Peng, Y.-F. Li, J. Mi, L. Yu, H.-Z. Huang, Reliability of complex systems under dynamic conditions: A bayesian multivariate degradation perspective, Reliability Engineering \& System Safety 153 (2016) 75 - 87. doi:https://doi. org $/ 10.1016 / j . r e s s .2016 .04 .005$. URL http://www.sciencedirect.com/science/article/pii/S0951832016300199

[15] Z. Tang, C. Zhou, W. Jiang, W. Zhou, X. Jing, J. Yu, B. Alkali, B. Sheng, Analysis of significant factors on cable failure using the cox proportional hazard model, IEEE Transactions on Power Delivery 29 (2) (2014) 951-957. doi: https://doi.org/10.1109/TPWRD.2013.2287025. URL https://ieeexplore.ieee.org/abstract/document/6661422/

[16] S. Lin, X. Sun, D. Feng, A failure rate model for traction transformer based on phm considering multiple factors, in: 2016 Prognostics and System Health Management Conference (PHM-Chengdu), 2016, pp. 1-6. doi:https://doi.org/ 10.1109/PHM. 2016.7819818.

URL https://ieeexplore.ieee.org/abstract/document/7819818/

[17] B. Ghodrati, U. Kumar, Reliability and operating environment-based spare parts estimation approach: A case study in kiruna mine, sweden, Journal of Quality in Maintenance Engineering 11 (2) (2005) 169-184. arXiv:https://doi. org/10.1108/13552510510601366, doi:10.1108/13552510510601366. URL https://doi.org/10.1108/13552510510601366

[18] A. Barabadi, Reliability and spare parts provision considering operational environment: A case study, International Journal of Perfomability Engineering 8 (2012) 497-506.

URL http://www.ijpe-online.com

[19] A. Barabadi, J. Barabady, T. Markeset, Application of reliability models with covariates in spare part prediction and optimization. a case study, Reliability Engineering \& System Safety 123 (2014) 1-7. doi:https://doi.org/10.1016/ j.ress.2013.09.012.

URL http://www.sciencedirect.com/science/article/pii/S095183201300269X

[20] M. Naseri, P. Baraldi, M. Compare, E. Zio, Availability assessment of oil and gas processing plants operating under dynamic arctic weather conditions, Reliability Engineering \& System Safety 152 (2016) 66 - 82. doi:https://doi. org $/ 10.1016 / j$.ress.2016.03.004.

URL http://www.sciencedirect.com/science/article/pii/S0951832016000703

[21] D. Kayrbekova, A. Barabadi, T. Markeset, Maintenance cost evaluation of a system to be used in arctic conditions: a case study, Journal of Quality in Maintenance Engineering 17 (4) (2011) 320-336. arXiv:https://doi.org/10.1108/ 13552511111180159 , doi:10.1108/13552511111180159. URL https://doi.org/10.1108/13552511111180159

545 [22] A. Barabadi, J. Barabady, T. Markeset, Maintainability analysis considering time-dependent and time-independent covariates, Reliability Engineering \& System Safety 96 (1) (2011) 210 - 217, special Issue on Safecomp 2008. doi: https://doi.org/10.1016/j.ress.2010.08.007.

URL http://www.sciencedirect.com/science/article/pii/S0951832010001924

[23] D. R. Cox, Models and life-tables regression, JR Stat. Soc. Ser. B 34 (1972) 187-220.

[24] L. Zhiguo, Z. Shiyu, S. Crispian, C. Suresh, Change detection in the cox proportional hazards models from different reliability data, Quality and Reliability Engineering International 26 (7) (2010) 677-689. arXiv:https: //onlinelibrary.wiley.com/doi/pdf/10.1002/qre.1132, doi:https://doi.org/10.1002/qre.1132. URL https://onlinelibrary.wiley.com/doi/abs/10.1002/qre.1132

[25] C. I. Bharat, K. Murray, E. Cripps, M. R. Hodkiewicz, Methods for displaying and calibration of cox proportional 
hazards models, Proceedings of the Institution of Mechanical Engineers, Part O: Journal of Risk and Reliability 232 (1) (2018) 105-115. doi:10.1177/1748006X17742779.

URL https://journals. sagepub.com/doi/abs/10.1177/1748006X17742779?journalCode=pioa

[26] A. Bendell, Proportional hazards modelling in reliability assessment, Reliability Engineering 11 (3) (1985) 175 - 183. doi:https://doi.org/10.1016/0143-8174(85)90070-8. URL http://www.sciencedirect.com/science/article/pii/0143817485900708

[27] Z. Chen, J. Ren, Y. Zhang, Z. Xing, Maintenance decision of door system based on phm-assisted rcm, in: Control Conference (CCC), 2017 36th Chinese, IEEE, 2017, pp. 7433-7437. doi : 10.23919/ChiCC.2017.8028530. URL https://ieeexplore.iee. org/document/8028530

[28] F. Wu, Y. Zhou, J. Liu, Modelling the effect of time-dependent covariates on the failure rate of wind turbines, in: Asset Intelligence through Integration and Interoperability and Contemporary Vibration Engineering Technologies, Springer, 2019, pp. 727-734. doi:10.1007/978-3-319-95711-1_71. URL https://link.springer.com/chapter/10.1007\%2F978-3-319-95711-1_71

[29] P. Mazidi, L. Bertling Tjernberg, M. A. Sanz Bobi, Wind turbine prognostics and maintenance management based on a hybrid approach of neural networks and a proportional hazards model, Proceedings of the Institution of Mechanical Engineers, Part O: Journal of Risk and Reliability 231 (2) (2017) 121-129. doi:10.1177/1748006X16686899. URL https://journals.sagepub.com/doi/abs/10.1177/1748006X16686899?journalCode=pioa

[30] Y. Shaban, S. Yacout, Predicting the remaining useful life of a cutting tool during turning titanium metal matrix composites, Proceedings of the Institution of Mechanical Engineers, Part B: Journal of Engineering Manufacture 232 (4) (2018) 681-689. doi:10.1177/0954405416654184. URL https://journals.sagepub.com/doi/abs/10.1177/0954405416654184?journalCode=pibb

[31] F. Ding, Z. He, Cutting tool wear monitoring for reliability analysis using proportional hazards model, The International Journal of Advanced Manufacturing Technology 57 (5-8) (2011) 565-574. doi:10.1007/s00170-011-3316-4. URL https://link.springer.com/article/10.1007\%2Fs00170-011-3316-4

[32] U. Leturiondo, O. Salgado, D. Galar, Estimation of the reliability of rolling element bearings using a synthetic failure rate, in: Current Trends in Reliability, Availability, Maintainability and Safety, Springer, 2016, pp. 99-112. doi: 10.1007/978-3-319-23597-4_8.

[33] R. Ahmad, S. Kamaruddin, I. A. Azid, I. P. Almanar, Failure analysis of machinery component by considering external factors and multiple failure modes-a case study in the processing industry, Engineering Failure Analysis 25 (2012) 182192. doi:10.1016/j.engfailanal.2012.05.007.

URL https://www.sciencedirect.com/science/article/abs/pii/S1350630712000908?via\%3Dihub

[34] L. Li, D. Ma, Z. Li, Cox-proportional hazards modeling in reliability analysis: A study of electromagnetic relays data, IEEE Transactions on Components, Packaging and Manufacturing Technology 5 (11) (2015) 1582-1589. doi:https: //doi.org/10.1109/TCPMT.2015.2480229.

URL https://ieeexplore.ieee.org/document/7298432/

[35] A. Tiwari, D. Roy, Estimation of reliability of mobile handsets using cox-proportional hazard model, Microelectronics Reliability 53 (3) (2013) 481 - 487. doi:https://doi.org/10.1016/j.microrel.2012.10.008. URL http://www.sciencedirect.com/science/article/pii/S0026271412004842

[36] Q. Xie, C. Bharat, R. N. Khan, A. Best, M. Hodkiewicz, Cox proportional hazards modelling of blockage risk in vitrified clay wastewater pipes, Urban Water Journal 14 (7) (2017) 669-675. arXiv:https://doi.org/10.1080/1573062X.2016. 1236135, doi:https://doi.org/10.1080/1573062X.2016.1236135. URL https://www.tandfonline.com/doi/abs/10.1080/1573062X.2016.1236135

[37] K. Xu, M. Xie, L. Tang, S. Ho, Application of neural networks in forecasting engine systems reliability, Applied Soft Computing 2 (4) (2003) 255 - 268. doi:https://doi.org/10.1016/S1568-4946(02)00059-5. URL http://www.sciencedirect.com/science/article/pii/S1568494602000595

[38] A. Marugan, F. Garcia Marquez, J. Pinar Perez, D. Ruiz-HernĀjndez, A survey of artificial neural network in wind energy systems, Applied Energy 228 (2018) 1822 - 1836. doi:https://doi.org/10.1016/j.apenergy.2018.07.084. URL http://www.sciencedirect.com/science/article/pii/S0306261918311048

[39] A. Z. Al-Garni, J. Ahmad, Artificial neural network application of modeling failure rate for boeing 737 tires, Quality and Reliability Engineering International 27 (2) (2010) 209-219. arXiv:https://onlinelibrary.wiley.com/doi/pdf/ 10.1002/qre.1114, doi:https://doi.org/10.1002/qre.1114. URL https://onlinelibrary.wiley.com/doi/abs/10.1002/qre.1114

[40] O. Fink, E. Zio, U. Weidmann, Predicting component reliability and level of degradation with complex-valued neural networks, Reliability Engineering \& System Safety 121 (2014) 198-206. doi : https://doi.org/10.1016/j.ress.2013. 08.004 .

610 URL http://www.sciencedirect.com/science/article/pii/S0951832013002391

[41] A. Beg, F. Awwad, W. Ibrahim, F. Ahmed, On the reliability estimation of nano-circuits using neural networks, Microprocessors and Microsystems 39 (8) (2015) 674-685. doi:https://doi.org/10.1016/j.micpro.2015.09.008. URL http://www.sciencedirect.com/science/article/pii/S0141933115001507

[42] T. Santhosh, V. Gopika, A. Ghosh, B. Fernandes, An approach for reliability prediction of instrumentation \& control cables by artificial neural networks and weibull theory for probabilistic safety assessment of npps, Reliability Engineering \& System Safety 170 (2018) 31 - 44. doi:https://doi.org/10.1016/j.ress.2017.10.010. URL http://www.sciencedirect.com/science/article/pii/S0951832017312152

[43] D. Faraggi, R. Simon, A neural network model for survival data, Statistics in Medicine 14 (1) (1995) 73-82. arXiv: https://onlinelibrary.wiley.com/doi/pdf/10.1002/sim.4780140108, doi:10.1002/sim.4780140108. URL https://onlinelibrary.wiley.com/doi/abs/10.1002/sim.4780140108

[44] T. Ching, X. Zhu, L. X. Garmire, Cox-nnet: An artificial neural network method for prognosis prediction of highthroughput omics data, PLOS Computational Biology 14 (4) (2018) 1-18. doi:10.1371/journal.pcbi.1006076. URL https://doi.org/10.1371/journal.pcbi.1006076

[45] A. Xiang, P. Lapuerta, A. Ryutov, J. Buckley, S. Azen, Comparison of the performance of neural network methods and cox regression for censored survival data, Computational Statistics \& Data Analysis 34 (2) (2000) $243-257$. doi:https://doi.org/10.1016/S0167-9473(99)00098-5.

URL http://www.sciencedirect.com/science/article/pii/S0167947399000985 
[46] E. Biganzoli, P. Boracchi, E. Marubini, A general framework for neural network models on censored survival data, Neural Networks 15 (2) (2002) 209 - 218. doi:https://doi.org/10.1016/S0893-6080(01)00131-9.

URL http://www.sciencedirect.com/science/article/pii/S0893608001001319

[47] R. M. Ripley, A. L. Harris, L. Tarassenko, Non-linear survival analysis using neural networks, Statistics in Medicine 23 (5) (2004) 825-842. arXiv:https://onlinelibrary.wiley.com/doi/pdf/10.1002/sim.1655, doi:https://doi.org/ $10.1002 / \operatorname{sim} .1655$.

URL https://onlinelibrary.wiley.com/doi/abs/10.1002/sim.1655

[48] P. Mazidi, M. Du, L. Bertling Tjernberg, M. Sanz Bobi, A health condition model for wind turbine monitoring through neural networks and proportional hazard models, Proceedings of the Institution of Mechanical Engineers, Part O: Journal of Risk and Reliability 231 (5) (2017) 481-494, cited By 3. doi:10.1177/1748006X17707902.

URL https://www.scopus.com/inward/record.uri?eid=2-s2.0-85029321409\&doi=10.1177\%2f1748006X17707902\& partnerID $=40 \&$ md5 $=97 \mathrm{e} 03777 \mathrm{e} 9 \mathrm{~d} 313 \mathrm{f} 9 \mathrm{e} 865 \mathrm{ecce} 4 \mathrm{fc} 40 \mathrm{eed}$

[49] J. F. Gomez, F. Olivencia, J. Ferrero, A. Crespo, G. Cerruela, Analysis of dynamic reliability surveillance: a case study, IMA Journal of Management Mathematics 29 (1) (2018) 53-67. arXiv:/oup/backfile/content_public/journal/ imaman/29/1/10.1093_imaman_dpw011/1/dpw011.pdf, doi:10.1093/imaman/dpw011.

[50] O. Fernando A., F. Jesus, G. Juan F., C. Adolfo, Failure mode prediction and energy forecasting of pv plants to assist dynamic maintenance tasks by ann based models, Renewable Energy 81 (2015) 227 - 238. doi:https://doi.org/10. $1016 / j$.renene.2015.03.023.

URL http://www.sciencedirect.com/science/article/pii/S0960148115002050

[51] A. Birolini, Reliability engineering, Vol. 5, Springer, 2007. doi:10.1007/978-3-662-03792-8. URL https://www.springer.com/gb/book/9783662037928

[52] A. Crespo, The maintenance management framework: models and methods for complex systems maintenance, 1st Edition, Relibaility Engineering, Springer Science \& Business Media, 2007. doi:https://doi.org/10.1007/ 978-1-84628-821-0.

URL https://link.springer.com/book/10.1007/978-1-84628-821-0\#about

[53] EN, 13306:2010. maintenance - maintenance terminology, Tech. rep., European Committee for Standardization (2010).

[54] A. Sharma, G. Yadava, S. Deshmukh, A literature review and future perspectives on maintenance optimization, Journal of Quality in Maintenance Engineering 17 (1) (2011) 5-25. doi:10.1108/13552511111116222. URL https://www. emeraldinsight.com/doi/abs/10.1108/13552511111116222? journalCode=jqme

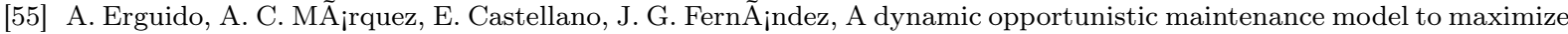
energy-based availability while reducing the life cycle cost of wind farms, Renewable Energy 114 (2017) $843-856$. doi:https://doi.org/10.1016/j.renene.2017.07.017.

URL http://www.sciencedirect.com/science/article/pii/S0960148117306304

[56] H. Abdollahzadeh, K. Atashgar, Optimal design of a multi-state system with uncertainty in supplier selection, Computers \& Industrial Engineering 105 (2017) 411 - 424. doi:https://doi.org/10.1016/j.cie.2017.01.019. URL http://www.sciencedirect.com/science/article/pii/S0360835217300384

[57] W. Zhu, M. Fouladirad, C. BÃ@Crenguer, A multi-level maintenance policy for a multi-component and multifailure mode system with two independent failure modes, Reliability Engineering \& System Safety 153 (2016) 50 - 63. doi: https://doi.org/10.1016/j.ress.2016.03.020.

URL http://www.sciencedirect.com/science/article/pii/S0951832016300102 


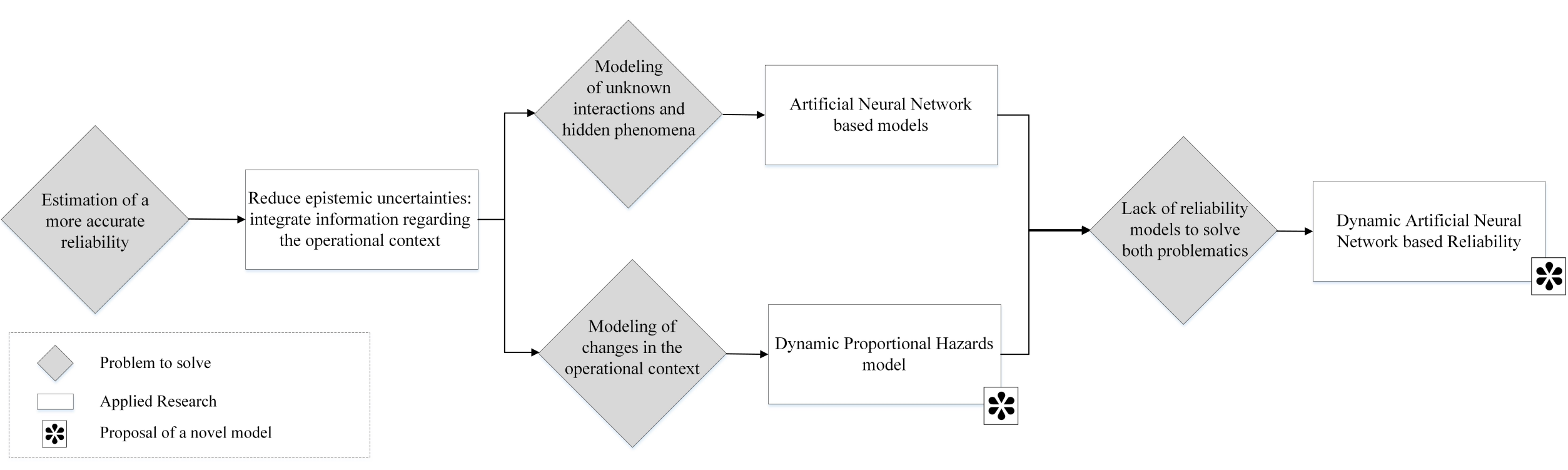




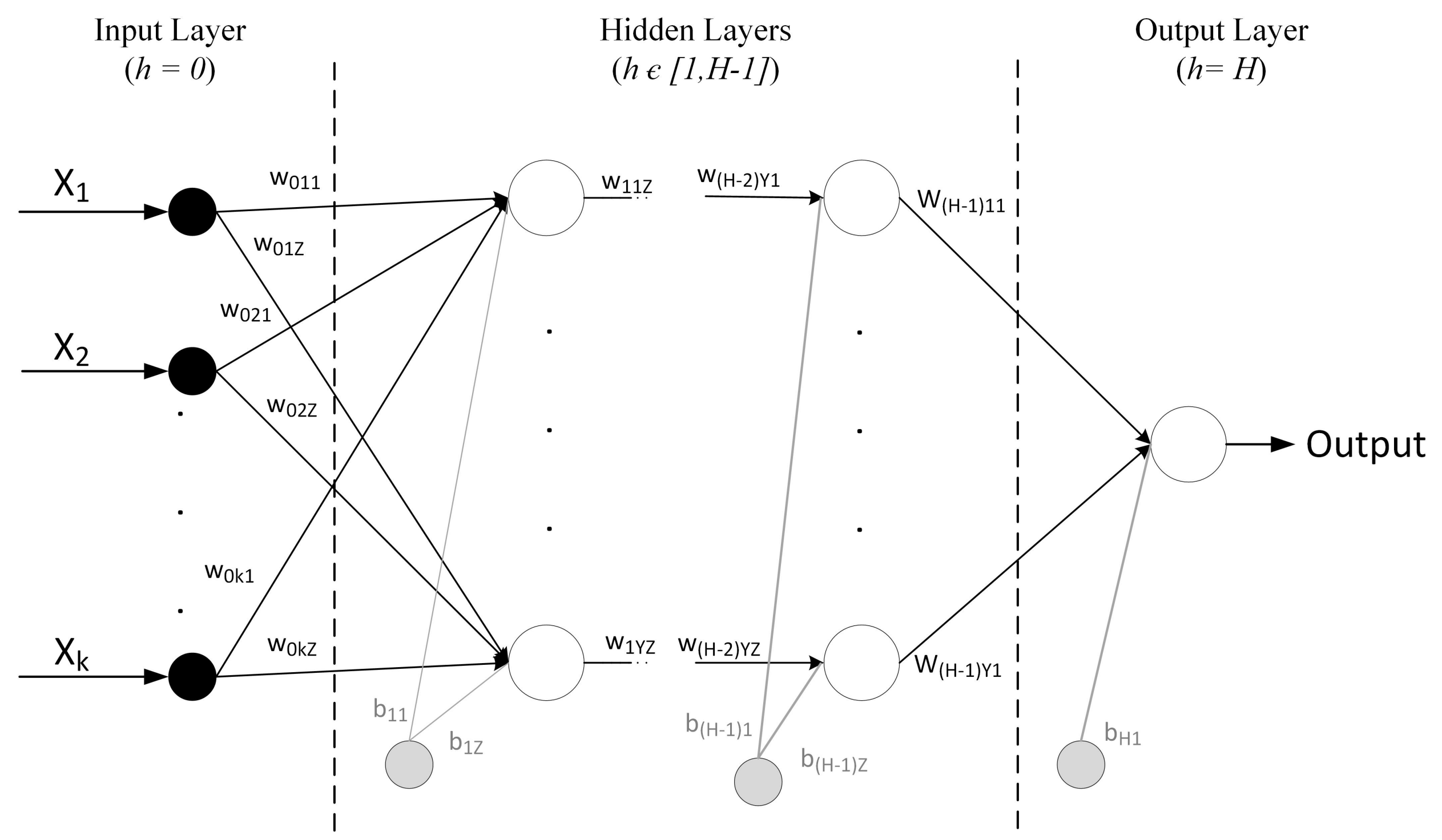




\section{OPC EPC}
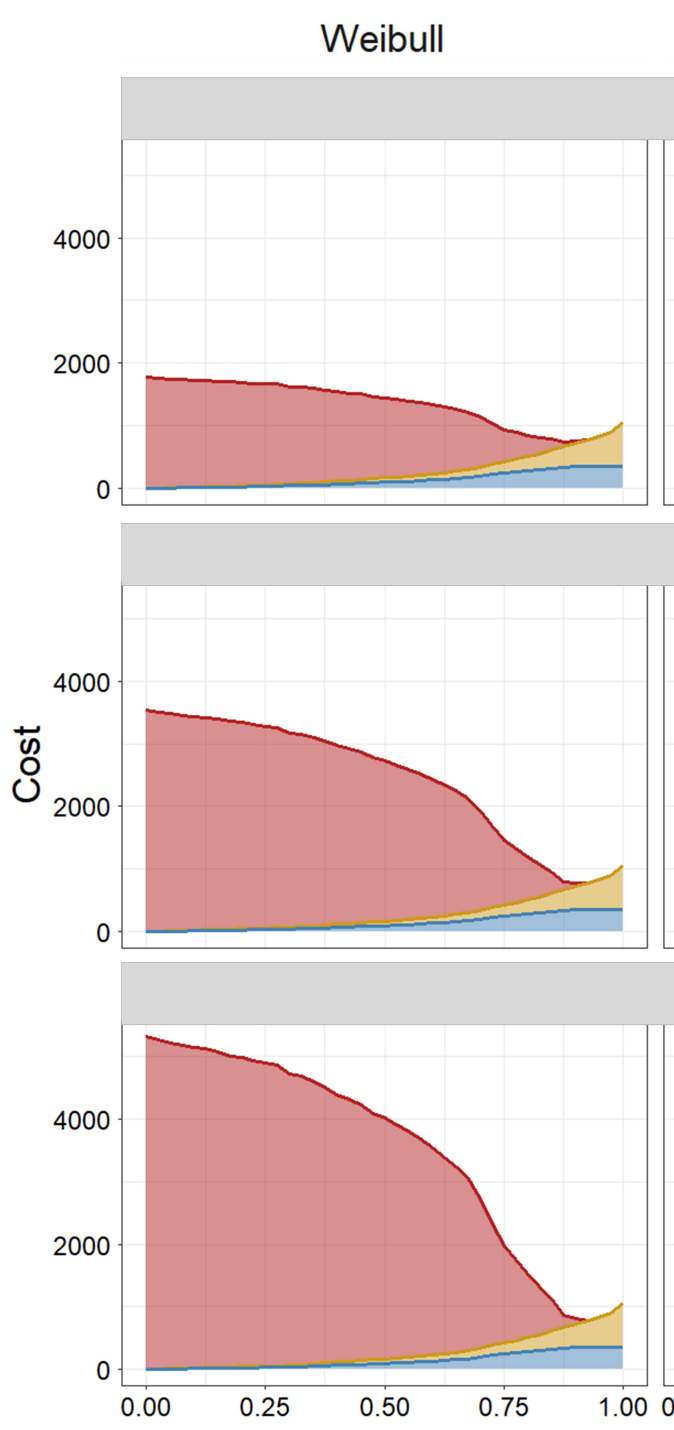

000.00

\section{Weibull PHM}

$\mathrm{MCR}=5$

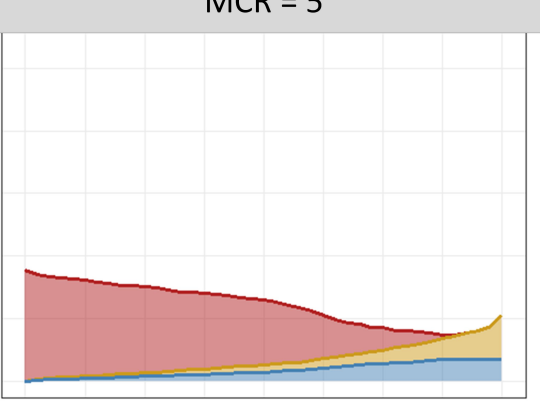

$\mathrm{MCR}=10$

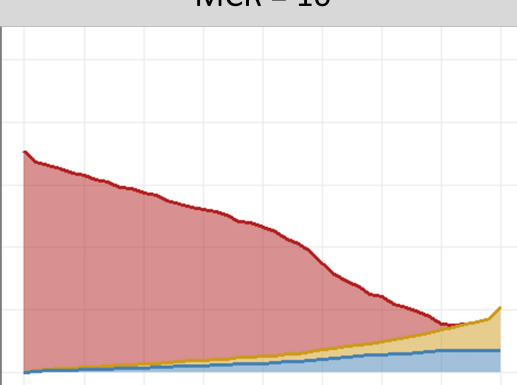

$\mathrm{MCR}=15$

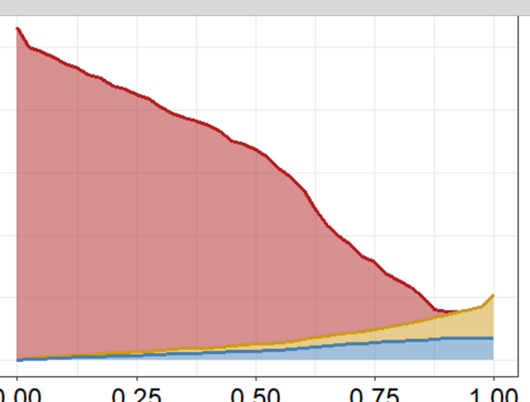

Threshold
Savings between

Savings between

Dynamic ANN-based

Dynamic ANN-based

\& Weibull

\& Weibull-PHM

Savings for $M C R=5$
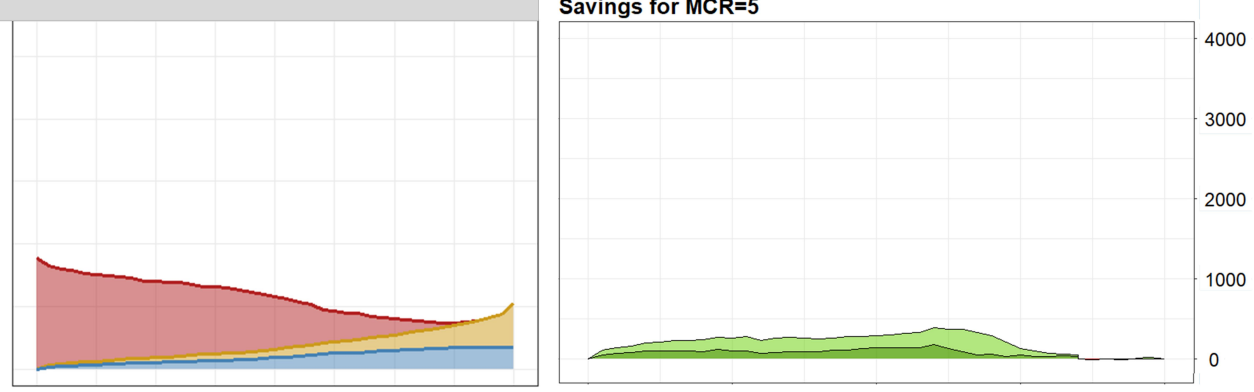

Savings for $M C R=10$
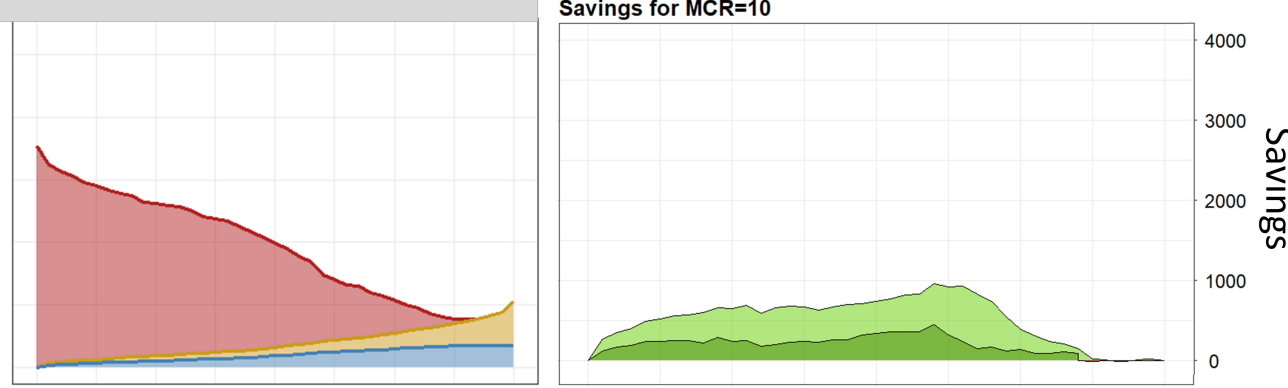

Savings for $M C R=15$
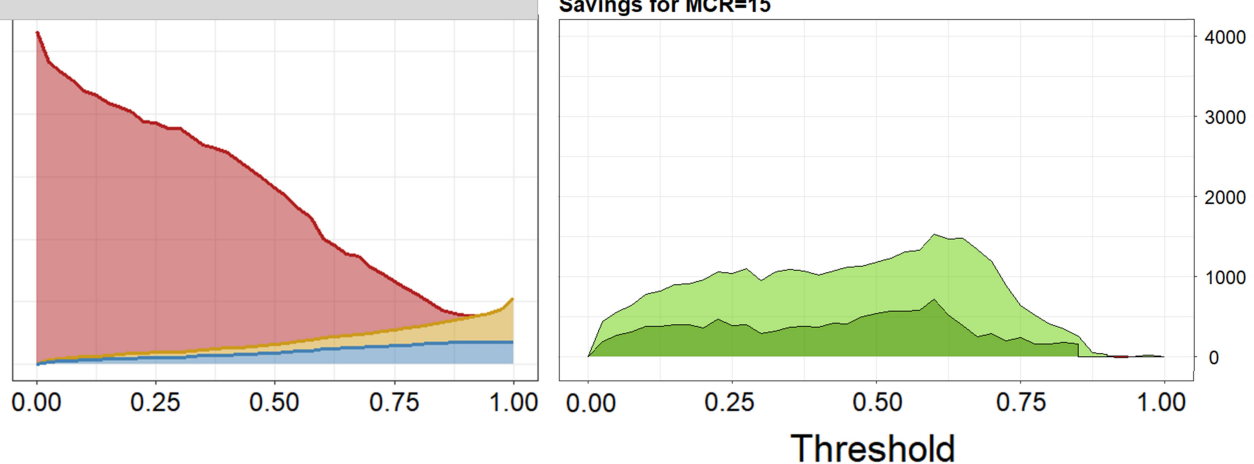